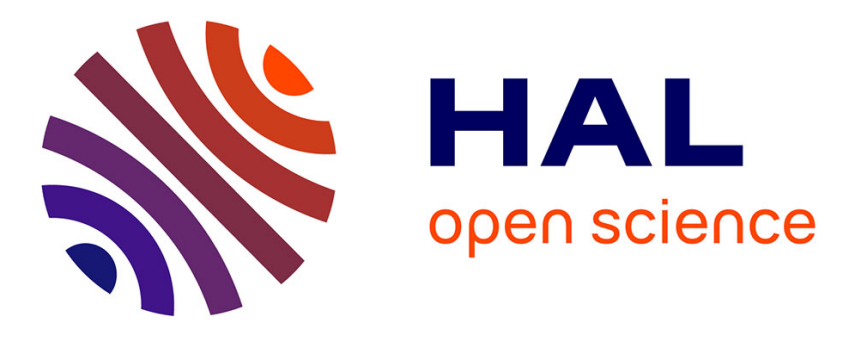

\title{
OLSR and WCPD as Basis for Service Discovery in MANETs
}

\author{
Tom Leclerc, Laurent Ciarletta, Adrian Andronache, Steffen Rothkugel
}

\section{To cite this version:}

Tom Leclerc, Laurent Ciarletta, Adrian Andronache, Steffen Rothkugel. OLSR and WCPD as Basis for Service Discovery in MANETs. The Second International Conference on Mobile Ubiquitous Computing, Systems, Services and Technologies - UBICOMM'08, 2008, Valencia, Spain. pp.184-190, 10.1109/UBICOMM.2008.20 . inria-00334479

\section{HAL Id: inria-00334479 \\ https://hal.inria.fr/inria-00334479}

Submitted on 15 Oct 2010

HAL is a multi-disciplinary open access archive for the deposit and dissemination of scientific research documents, whether they are published or not. The documents may come from teaching and research institutions in France or abroad, or from public or private research centers.
L'archive ouverte pluridisciplinaire $\mathbf{H A L}$, est destinée au dépôt et à la diffusion de documents scientifiques de niveau recherche, publiés ou non, émanant des établissements d'enseignement et de recherche français ou étrangers, des laboratoires publics ou privés. 


\section{OLSR and WCPD as Basis for Service Discovery in MANETs}

\author{
Tom Leclerc, Laurent Ciarletta \\ LORIA, Nancy, France \\ \{tom.leclerc,laurent.ciarletta\}@loria.fr
}

\author{
Adrian Andronache, Steffen Rothkugel \\ University of Luxembourg, Luxembourg \\ \{adrian.andronache, steffen.rothkugel\}@uni.lu
}

\begin{abstract}
Service discovery is one of the most fundamental building blocks of self-organization. While mature approaches exist in the realm of fixed networks, they are not directly applicable in the context of MANETs. We investigate and compare two different protocols as basis for service discovery, namely OLSR and WCPD. OLSR is a proactive routing protocol while WCPD is a path discovery protocol integrating node and link stability criteria.

Two conflicting objectives of service discovery are the coverage of service queries together with the required bandwidth. Simulations are performed based on a setting in a city center with human mobility. We show that OLSR outperforms WCPD in terms of coverage. Due to its proactive nature, however, bandwidth consumption is high. WCPD on the other hand is much more bandwidth efficient, but at the cost of lower coverage.

Finally, we motivate employing OLSR on top of an overlay topology maintained by WCPD. This fosters stability while reducing overhead and keeping coverage high.
\end{abstract}

\section{Introduction}

In this paper we consider large Mobile Ad hoc NETworks (MANET) where the wirelessly connected devices communicate without any infrastructure with each other. In order to provide ad hoc networks with useful, user friendly and interesting features service discovery should be provided. Service discovery facilitates resource/data/multimedia sharing or for example ad hoc/situated games, furthermore it permits to take full advantage of the dynamic networks specificities.

The goal of service discovery is mainly to find services provided by other nodes in the network in an automated way and use them by knowing a basic set of information. Initially, service discovery protocols were designed for wired networks and most services were simple services, like for instance printing services. Not every node can or wants to achieve a given service. For example to print, a node doesn't need to be connected directly to the printer, just by using the service provided by the node that is actually connected to the printer is enough to be able to print. In the last years a wide range of services became popular, like music sharing, game services or gateway services providing Internet access. Without infrastructure, as in ad hoc networks, the need to automatically, hence not manually which would be to complicated, discover services, that the network offers is even more crucial than in classical wired networks as no central information is available. Service discovery is even more indispensable for nodes with limited capabilities, which want to use a service without having the capability to host or run it by themselves. In ad hoc networks nodes, and the services they provide, can come and go so that topology changes all the time. These topological changes have to be reflected on the service discovery architecture.

In wired network a service failure is mostly due to a service inherent problem while in ad hoc networks topology causes most of the service failures.

In mobile ad hoc networks, just finding a service that suits best the user's and application's requirements is merely sufficient. In today's service-rich and growing networks, what matters is finding the best service that also optimizes part or all of the following elements: the hop distance, stability, availability, effectiveness, etc. To enable these requirements a topological structure seems imperative.

We consider topology oriented protocols where some nodes have higher responsibilities like for instance relaying, grouping or disseminating messages from other nodes. Taking the topology building techniques from these protocols for service discovery protocols, allows us to have an efficient dissemination of service information and enables us to take advantage of the higher responsibility nodes (also called directories in service discovery) which store, forward or query service information for other nodes.

In this paper we investigate and compare the performances of the two topology conscious protocols OLSR [1] and WCPD [2], in regards to their topology 
architecture, for service discovery achievements. As the capabilities of the devices in ad hoc networks are always growing but still heterogeneous, from low capacities to very high, we consider a full range of services from simple classical printing services to advanced multimedia services.

\section{Related work}

As stated before, most of the service discovery protocols designed for wired network, like SLP [3], JINI [4], or UPnP [5] do not take into account any topology information.

Several discovery mechanisms can be implemented and mixed in service discovery protocols: active/passive discovery, directory or directory-less discovery. Active discovery means nodes broadcast a request for a service in the network and receive one or more answer from the service provider matching the request. Passive discovery means service providers periodically announce their services to all the nodes in the network. To reduce broadcasting in the network from many nodes, eventually resulting in massive flooding of the network, directory nodes are used. These nodes are elected by the surrounding nodes and are responsible for the electing set of nodes. Once elected, they store service announcements and corresponding service information, handle queries of their "slaves", hence reducing considerably the load of the network and the non-directory nodes.

Allia [6] is a peer-to-peer caching based and policydriven service discovery framework. It removes the leader election problem by enabling every node to be self-sufficient. Every node creates alliances with other nodes and uses local policies for forward and caching decisions. A node knows which nodes are in his alliance, but it does not know in which alliances it is included from other nodes. As Allia does not take into account the network topology it does not fit our previously stated requirements.

Others propose to take partial aspects of the topology into account like in [7] and [8], where both use a multicast topology for the service discovery which is given by the network layer. Unfortunately the use of multicast induces a large number of control messages, which also does not suit our requirements.

The most interesting approaches for our work are the ones that take advantage of network topology to disseminate service information efficiently.

OLSR (Optimized Link State Routing) is well known as an ad hoc routing protocol but it is also a popular choice for service discovery architectures, mainly as an underlying connectivity provider. In [9] and [10] the OLSR protocol is used to encapsulate the service discovery messages. Also in Scalable Service Discovery or MANET [11] the bordercasting, which is the "Multipoint Relay (MPR)" mechanism of OLSR, is used to efficiently flood the network.

Another interesting architecture is the Hierarchical OLSR [12] (HOLSR) which actually is not a service discovery protocol, but does address our problem of disseminating information through ad hoc networks efficiently.

The other type of topology we are taking into consideration is the cluster topology. Although in service discovery the cluster topology can be referred as service discovery with directory. The service discovery directories correspond to the clusterheads of the cluster architecture. Directories are elected on various criteria, like for instance node coverage.

A good example is Scalable Service Discovery for MANET [11] which is a distributed central directories discovery architecture. Directories are responsible for caching the service descriptions, advertising their presence to nodes within their vicinity and handling their service requests by checking the local cache or forwarding the query to other directories. The election of the directories is done on the fly and the main election criterion is the node coverage. To exchange the directory profiles they use bloom filters and "bordercast" (using MPRs [1]) it in the two-hop neighborhood. The selection of the directory nodes relies on the node coverage which can be a problem if for example a nearby coming node traverses the network has a big node coverage at one particular moment but will, after being elected, disconnect shortly because of his mobility.

\section{Topology protocols}

This section briefly describes the protocols, OLSR and WCPD used in our experiments to find a good suited topology for service discovery. We choose to compare OLSR and WCPD because both build well known topology architectures. On one hand OLSR builds a tree topology and on the other hand WCPD builds a star topology.

\subsection{OLSR}

The Optimized Link State Routing Protocol (OLSR) is a well known routing protocol designed for ad hoc networks. It is a proactive protocol; hence it periodically exchanges topology information with other nodes of the network. One-hop neighborhood and two-hop neighborhood are discovered using Hello 
Messages (similar to the beacon message). The multipoint relay (MPR) nodes are calculated by selecting the smallest one-hop neighborhood set needed to reach every two-hop neighbor node. The topology control information is only forwarded by the nodes which are selected as MPR. Every node possesses then a routing table containing the shortest path to every node of the network. OLSR enables efficient flooding of the network by building a Tree like topology for every node from a source (Figure 1).

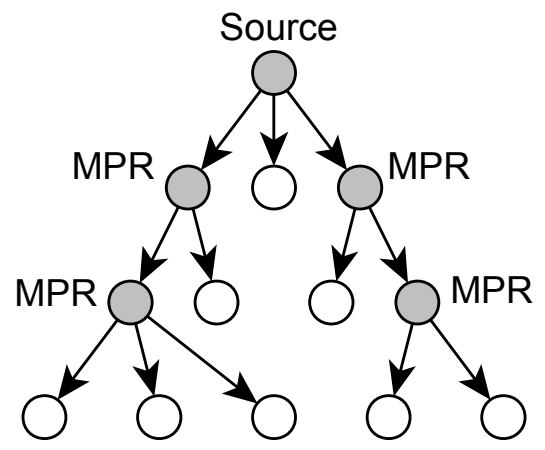

Figure 1. OLSR topology for one source node in particular.

\subsection{WCPD}

The Weighted Cluster-based Path Discovery protocol (WCPD) is designed to take advantage of the cluster topology build by Node and Link Weighted Clustering Algorithm (NLWCA) [13] in order to provide reliable path discovery and broadcast mechanisms in mobile ad hoc networks (Figure 2).

NLWCA organizes ad hoc networks in one hop clusters by using only information available locally. Each device elects exactly one device as its clusterhead, i.e. the neighbor with the highest weight.

The main goal of the algorithm is to avoid superfluous re-organization of the clusters, particularly when clusters cross each other. To achieve this, NLWCA assigns weights to the links between the own node and the network neighbor nodes. This weight is used to keep track of the connection stability to the one-hop network neighbors. When a link weight reaches a given stability threshold the link is considered stable and the device is called stable neighbor device. The clusterhead is elected only from the set of stable neighbors which avoids the reorganization of the topology when two clusters are crossing for a short period of time.

WCPD discovers nearby stable-connected clusters in a pro-active fashion. For the nearby clusterheads discovery algorithm, WCPD uses the beacon, which is a periodically broadcasted message used in ad-hoc networks to detect devices in communication range.

WCPD runs on each network node and requires solely information available locally in the one hop neighborhood. The algorithm uses information provided by NLWCA: the set of stable connected network neighbor nodes and the ID of the own clusterhead. NLWCA also propagates by beacon the own weight and the ID of the current clusterhead.

Besides the information provided by NLWCA, the WCPD protocol uses the beacon to disseminate the list of locally discovered nearby connected clusterheads.

By doing so, every node has the following information about each stable one hop neighbor: its clusterhead ID and the ID set of discovered clusterheads and the respective path length. After the data of all stable one hop neighbors is checked, the set of discovered nearby clusterheads and the path length is inserted into the beacon in order to propagate it to the one hop neighborhood.

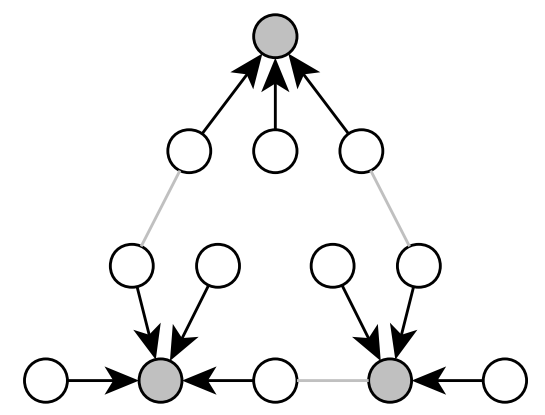

Figure 2. WCPD cluster topology. The clusterheads are connected by multi-hop paths, which are used for inter-cluster information exchange.

The WCPD broadcasting algorithm is simple and easy to deploy: the broadcast source node sends the message to the clusterhead, which stores the ID of the message and broadcasts it to the one hop neighborhood. After that, it sends it to all nearby clusterheads by multi-hop unicast and to the own subheads by unicast. The inter-cluster destination nodes repeat the procedure except that the message source clusters are omitted from further forwarding. Additionally the information about the ID of the broadcast messages and their sources is stored for a given period of time to avoid superfluous re-sending of the message.

The protocol sends the broadcast message to nearby clusters connected by stable links in order to disseminate it to the network partition. Nevertheless 
the message also reaches crossing clusters since the broadcasts are received by all nodes in the one-hop neighborhood of local leaders. This increases the chance that the message reaches a high number of nodes in the mobile network partition.

\section{Experiments}

In order to determine the best suited topology for our service discovery protocol, we implemented both protocols on the top of the JANE simulator [14] and performed several experiments.

\subsection{Simulation settings}

For the conducted experiments we used the Restricted Random Way Point mobility model [15], whereby the devices move along defined streets on the map of Luxembourg City for 5 minutes (Figure 3). For each device the speed was randomly varied between $[0.5 ; 1.5]$ units/s. At simulation startup, the devices are positioned at random selected crossroads and the movement to other crossroads is determined by the given random distribution seed. For the experiments a number of ten different random distribution seeds were used in order to feature results from different topologies and movement setups.

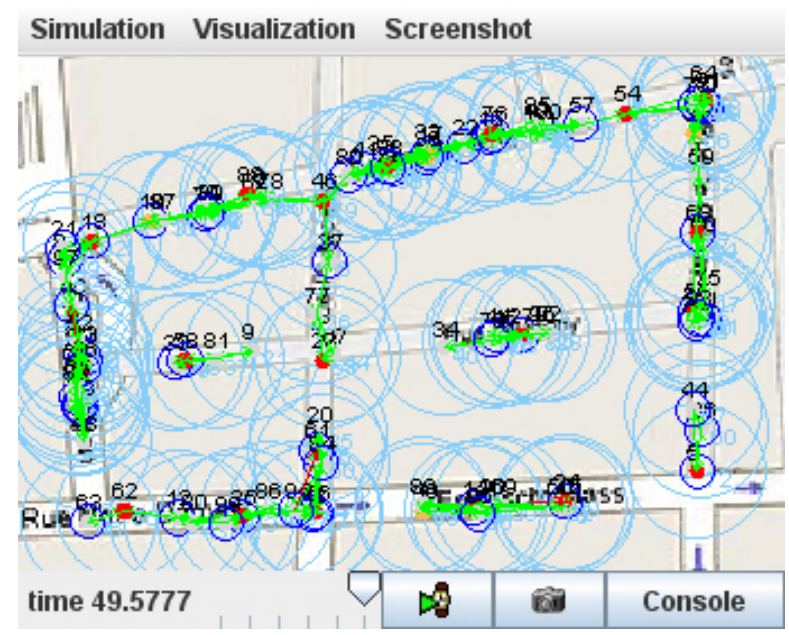

Figure 3. JANE simulating the protocols on 100 devices. The mobile devices move on the streets of the Luxembourg City map. The devices move with a speed of $0.5-1.5 \mathrm{~m} / \mathrm{s}$

For the used mobile environment where nodes move with low speeds between 1.8 and $5.4 \mathrm{~km} / \mathrm{h}$ the NLWCA link-stability threshold is set on 2.
Simulations were done to determine both the used bandwidth in order to build the topologies and the information dissemination performance of broadcasting on top of the two different topologies.

To build the MPR topology, OLSR exchanges the sets of one-hop neighbor nodes with every node in the communication range. Similar to OLSR, WCPD use the beacon to exchange the list of the discovered nearby-clusterheads with the one-hop neighbor nodes. To find out the network load produced during this phase, the size of both the one-hop neighbor sets and the size of discovered clusterheads were tracked every second of the simulation.

In order to monitor the information dissemination performance and network load of the broadcasting mechanisms, a node was chosen to broadcast a message every 10 seconds during different simulation runs using different distribution seeds. The number of sent messages (i.e. broadcasts and unicasts) during the dissemination and the number of reached network nodes were tracked.

\subsection{Results}

The results in figures 4, 5 and 6 are illustrating the size of the exchanged node-ID lists at the respective point in the timeline. To calculate the bandwidth used by the protocol, one needs to take into consideration the time interval used to periodically send the exchange messages (i.e. hello messages or beacons) and the size of the used node IDs (e.g. 32 bits for IPv4 addresses). This leads to formula (i) for a mean bandwidth B used in an IPv4 network where $|\mathrm{S}|$ is the mean number of exchanged addresses and $t$ is the time between the periodically exchanges:

$$
B=\frac{|S| \times 32}{t} \mathrm{bits} / \mathrm{sec}
$$

The results illustrated in figures 4,5 and 6 show that OLSR uses a higher bandwidth in both sparser and denser networks. This situation was expected since OLSR is exchanging the set of one-hop neighbors needed for the MPR nodes election.

In contrast to OLSR, WCPD only exchange the set of local discovered nearby clusterhead and sub-heads in order to discover stable paths between clusters in the network vicinity. The NLWCA protocol elects one clusterhead/sub-head in each one-hop neighborhood, which means that the number of clusterheads is a fractional amount of the number of nodes in the network. 


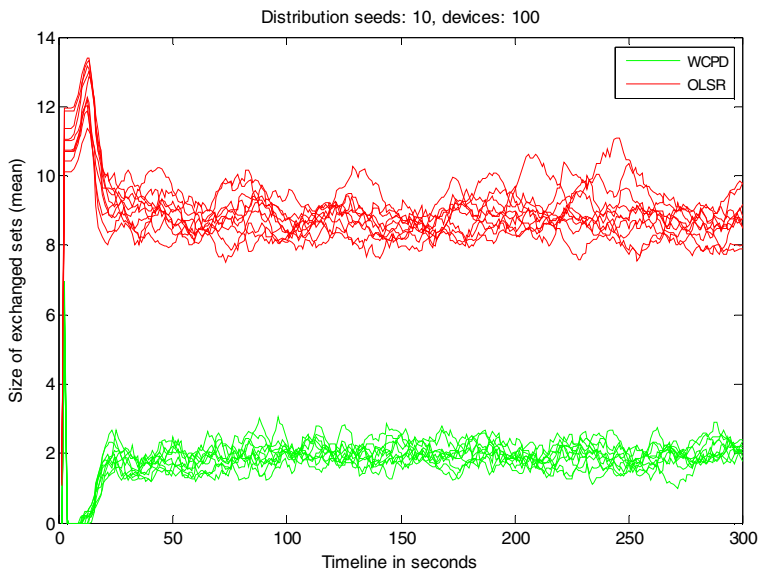

Figure 4. Size of the sets exchanged per second in order to build the topology.

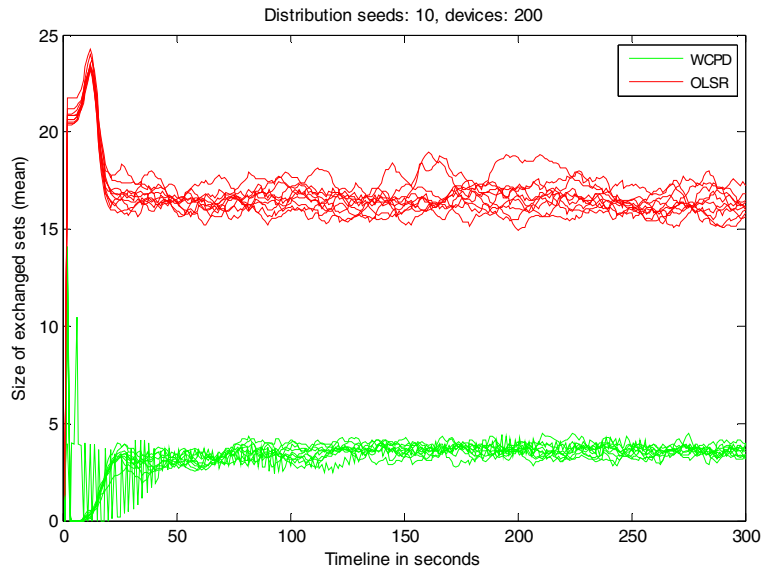

Figure 5. Size of the sets exchanged per second in order to build the topology.

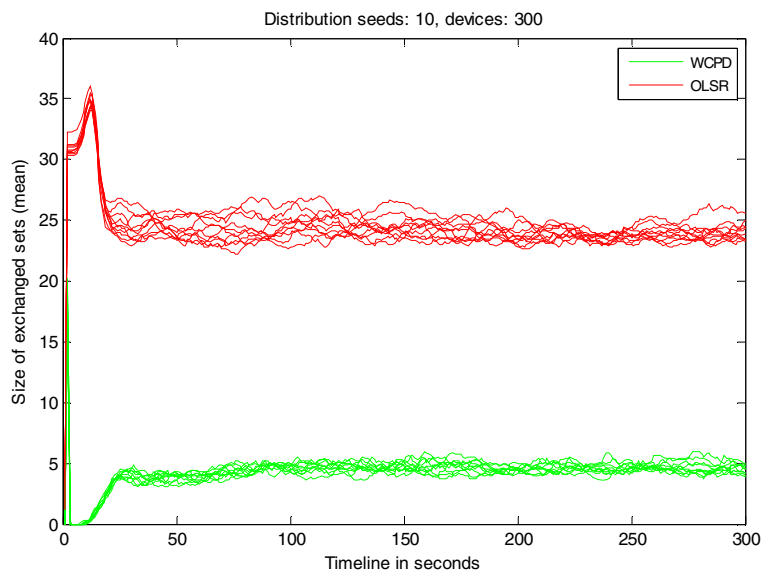

Figure 6. Size of the sets exchanged per second in order to build the topology.

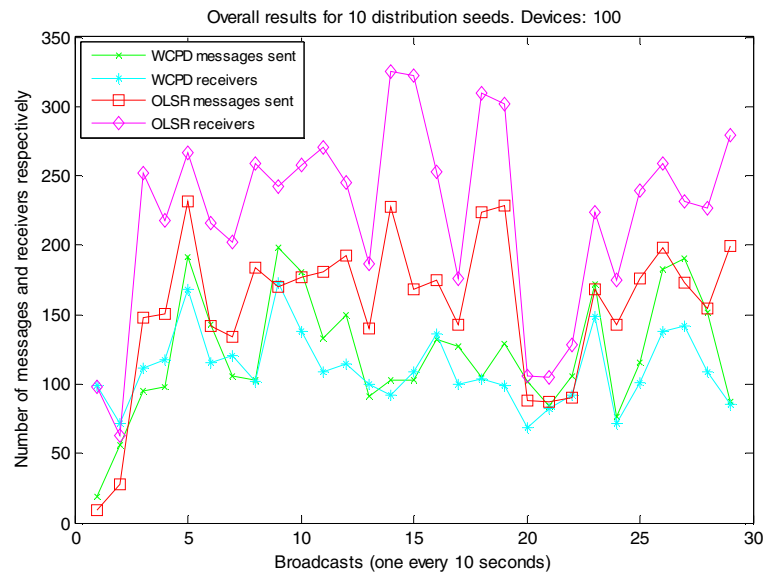

Figure 7. Overall number of sent messages and node receivers respectively for 100 nodes.

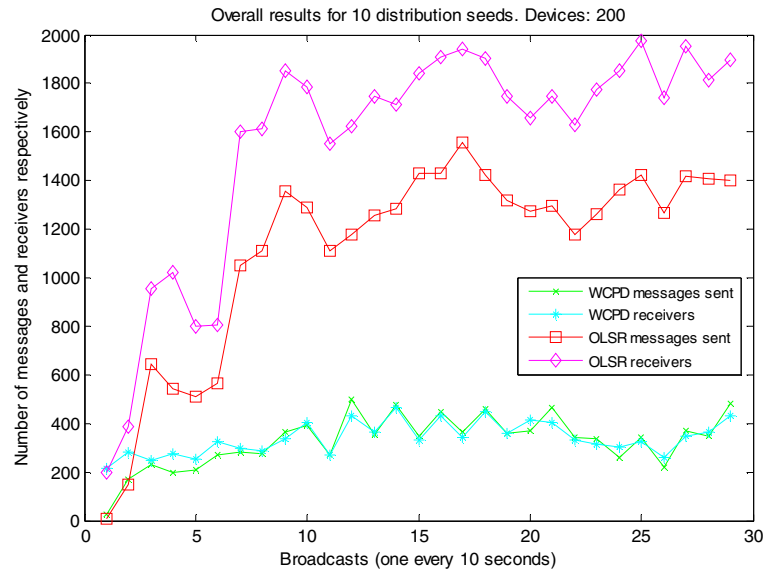

Figure 8. Overall number of sent messages and node receivers respectively for $\mathbf{2 0 0}$ nodes.

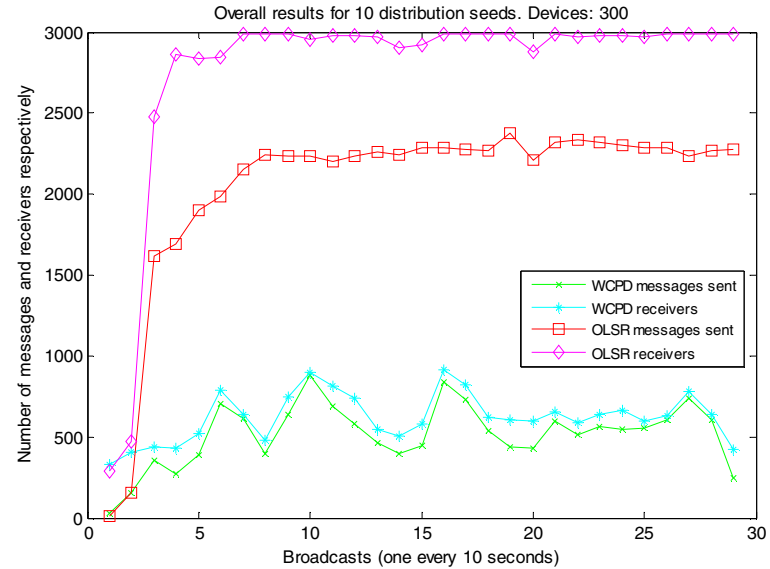

Figure 9. Overall number of sent messages and node receivers respectively for $\mathbf{3 0 0}$ nodes. 
The tracking results regarding the message dissemination performance and network load of the broadcasting protocols are presented in figures 7,8 and 9. The overall results show that the broadcasting on top of the OLSR topology performs much better in terms of message dissemination than on top of the WCPD topology. The denser the network, the higher is the difference between both the number of sent messages and the number of receiver nodes.

\section{Future work: A hybrid approach}

OLSR broadcasting is based on flooding the network in an efficient way via the MPRs such that messages reach all nodes already captured. Even in the presence of mobility, the broadcast will arrive at a high number of nodes. In contrast to that, the WCPD approach aims at spreading the messages between topology structures that are considered to be connected in a stable way. Especially in the presence of mobility, the stability threshold might not be reached by all nodes, which might result in a smaller number of broadcast receivers.

We propose to overlay both topologies - in this context for service discovery-by employing the OLSR MPR algorithm on top of the WCPD cluster topology.

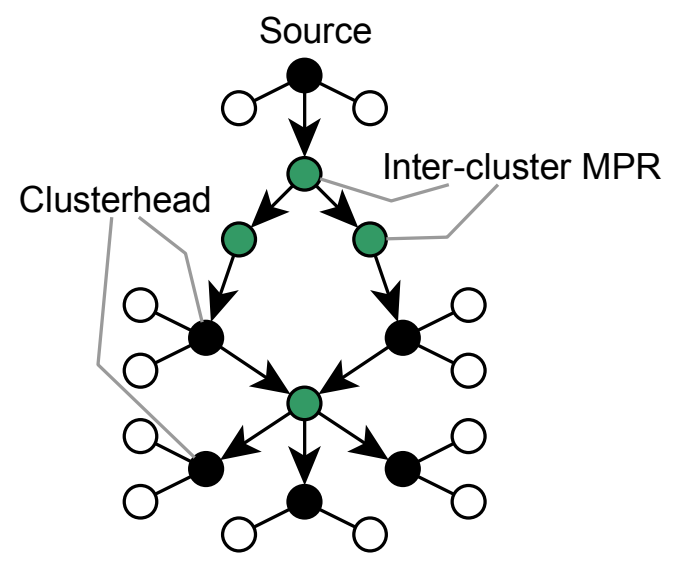

Figure 10. A hybrid architecture where the OLSR MPR protocol is used to connect nearby clusters discovered by the WCPD protocol.

In this hybrid approach clusterheads are used as service discovery directories. The discovery of nearby directories in turn is facilitated and maintained by the WCPD protocol. The communication paths between the directories used to exchange service discovery information are maintained by OLSR. Thus, the OLSR protocol has to establish the MPR topology only between clusterheads, which dramatically reduces the required communication load. Additionally, the OLSR topology on top of the cluster topology will result in optimized inter-cluster communication paths.

\section{Conclusion}

The simulation results show that between the two analyzed approaches the one based on OLSR is the better choice in order to reach as many nodes as possible by broadcasting for instance service discovery queries. This protocol highly outperforms in terms of broadcast receivers the WCPD approach that fosters the communication between nearby clusters considered to be stable-connected. On the other side, the network load produced by OLSR to build the topology is much higher compared to the one of the WCPD protocol. Besides that, services discovered on nodes in the network vicinity are more valuable than the ones on nodes topologically far away. The multi-hop path to a service host can be easily lost in mobile environments due to the movement of the nodes or network partitioning.

In conclusion the OLSR broadcasting approach has the advantage of reaching a much higher number of nodes than WCPD but at the cost of high network overload for the topology maintenance.

In future work we aim to combine the two protocols in a synergetic way by building clusters of stable connected nodes and using the OLSR topology on top of the cluster topology. Thus, a better inter-cluster path discovery and loop-free broadcasting mechanism may be provided at a low network load used for topology maintenance. This will enable the service discovery protocol to take advantage of stable paths to service hosts in the vicinity and at the same time to reach a high number of network nodes by broadcast.

\section{References}

[1] T. Clausen, P. Jacquet, et. al., "Optimized Link State Routing Protocol", RFC 3626, http://ietf.org/rfc/rfc3626.txt, October, 2003.

[2] A. Andronache and S. Rothkugel, "HyTrace-Backboneassisted Path Discovery in Hybrid Networks", International Conference on Communication Theory, Reliability, and Quality of Service, CTRQ 2008, Bucharest, Romania, July 2008

[3] E. Guttman, "Service Location Protocol: Automatic discovery of IP network services", IEEE Internet Computing, 3(4), July1999.

[4] Sun Microsystems: JiniTechnology Core Platform Specification. Version 2.0, 2003 
[5] UPnP Forum: Understanding Universal Plug and Play White Paper, http://www.upnp.org, 2000

[6] O. Ratsimor, et al., "Allia: Alliance-based service discovery for ad-hoc environments", ACM Mobile Commerce Workshop, 2002

[7] S. Helal, et. al., "Konark - A Service Discovery and Delivery Protocolfor Ad-hoc Networks", Proceedings of the Third IEEE Conference on Wireless Communication Networks (WCNC), New Orleans, 2003

[8] Ulas C. Kozat and Leandros Tassiulas, "Service discovery in mobile ad hoc networks: an overall perspective on architectural choices and network layer support issues", Ad Hoc Networks, January 2004, Vol. 2, No. 1., pp. 23-44.

[9] L. Li and L. Lamont, "A Lightweight Service Discovery Mechanism for Mobile Ad hoc Pervasive Environment using Cross-Layer Design", In Proceedings of the 2nd Mobile Peer-to-Peer Computing Workshop (MP2P), in conjunction with the 3rd IEEE International Conference on Pervasive Computing and Communications (PerCom'05), Hawaï, march 2005.

[10] J.L. Jodra, et. al., "Service discovery mechanism over OLSR for mobile ad-hoc networks", AINA2006, 20th International Conference on Volume 2, April 2006, pp. 6.

[11] F. Sailhan, V. Issarny, "Scalable Service Discovery for MANET", Proceedings of the 3rd IEEE Int'l Conf. on Pervasive Computing and Communications, 2005

[12] L. Villasenor-Gonzalez, Ying Ge, L. Lament, "HOLSR: a hierarchical proactive routing mechanism for mobile ad hoc networks". Communications Magazine, IEEE Volume 43, Issue 7, July 2005, pp. 118-125

[13] Adrian Andronache, Steffen Rothkugel, "NLWCANode and Link Weighted Clustering Algorithm for Backbone-Assisted Mobile Ad Hoc Networks",ICN2008, 2008, pp. 460-467

[14] Daniel Görgen, Hannes Frey and Christian Hiedels, "JANE - The Java Ad-hoc Network simulation and evaluation Environment", In Proceedings of the 40th Annual Simulation Symposium (ANSS 2007), Norfolk, VA, USA, 2007.

[15] Ljubica Blazevic, Silvia Giordano, Jean-Yves Le Boudec, "Self Organized Terminode Routing", Cluster Computing, Vol.5, No. 2, April 2002 